TRANSACTIONS OF THE

AMERICAN MATHEMATICAL SOCIETY

Volume 358, Number 8 , Pages 3623-3635

S 0002-9947(05)03809-2

Article electronically published on December 20, 2005

\title{
ON CARTAN MATRICES AND LOWER DEFECT GROUPS FOR COVERING GROUPS OF SYMMETRIC GROUPS
}

\author{
CHRISTINE BESSENRODT AND JØRN B. OLSSON
}

To our friend Alun Morris on the occasion of his 70th birthday

\begin{abstract}
We determine the elementary divisors of the Cartan matrices of spin $p$-blocks of the covering groups of the symmetric groups when $p$ is an odd prime. As a consequence, we also compute the determinants of these Cartan matrices, and in particular we confirm a conjecture by Brundan and Kleshchev that these determinants depend only on the weight but not on the sign of the block.
\end{abstract}

The main purpose of this paper is to determine the elementary divisors of the Cartan matrices of spin blocks in odd characteristic $p$ of the covering groups $\hat{S}_{n}$ of the symmetric groups. It is known that the invariant factors of the Cartan matrix of a $p$-block $B$ of a finite group $G$ are in fact the orders of the defect groups of certain $p$-regular conjugacy classes which are associated to $B$ in a so-called "block splitting" of the conjugacy classes of $G$. These defect groups are referred to as "lower defect groups" for $B$ and thus it is possible to compute the elementary divisors of the Cartan matrix of $B$ by determining the lower defect group multiplicities $m_{B}^{(1)}(Q)$ for $p$-subgroups $Q$ of $G$ ([4], 12]). We use this method in the present paper.

The corresponding question for the symmetric groups was studied in [13]. The computations there were eased by the simplicity of the subpair structure in $S_{n}$. In $\hat{S_{n}}$ the situation is considerably more complicated [7. Thus for our work here we also need extensions of the existing general results. In particular, Section 2 below may be relevant outside the concrete questions about $\hat{S}_{n}$ at hand.

The paper is organized as follows. In Sections 1 and 2 a general class of groups is studied which includes the covering groups of symmetric and alternating groups and their $p$-local subgroups. Here the new Proposition 2.3 is of particular importance. It is applied in Section 3 where we study spin blocks and doubling classes in $\hat{S_{n}}$. As in 13 the multiplicity of 1 as a lower defect group plays a key rôle, as it is always a factor of the general multiplicities. The purpose of Section 4 is then to compute this multiplicity of 1 in terms of the weight and sign of a spin block. It turns out that only the multiplicity of 1 is dependent on the sign of the block. The multiplicities of non-trivial $p$-subgroups are sign independent. As a consequence we are able to confirm a conjecture by Brundan and Kleshchev [6, p. 434] that the determinant of the Cartan matrix of a spin block is also independent of the sign (type) of the

Received by the editors December 15, 2003 and, in revised form, August 18, 2004 and August 20, 2004.

2000 Mathematics Subject Classification. Primary 20C30; Secondary 20C25.

This work was partially supported by The Danish National Research Council.

(C) 2005 by the authors 
block. In the final section a number of generating functions are determined, from which the multiplicities of lower defect groups for spin blocks and the determinants of the Cartan matrices may be computed explicitly.

\section{SPIN CHARACTERS AND DOUBLING ClASSES}

We want to describe the lower defect group multiplicities in $\hat{S}_{n}$. Since some of these are locally determined, we need to consider a more general situation, along the lines of 8 .

We consider the class $\mathcal{G}$ of finite groups $\hat{G}$ with a central subgroup $Z=\langle z\rangle$ of order 2 and a "sign" homomorphism $s: \hat{G} \rightarrow\{+1,-1\}$ with $s(z)=1$. Let $G$ denote the quotient group $\hat{G} / Z$, and let $\pi=\pi_{G}$ be the canonical epimorphism from $\hat{G}$ to $G$. We denote the kernel of $s$ by $\hat{H}$, and set $H=\hat{H} / Z$. Thus $|G: H| \leq 2$.

Our main example is of course $(\hat{G}, G, \hat{H}, H)=\left(\hat{S_{n}}, S_{n}, \hat{A_{n}}, A_{n}\right)$.

Let $\hat{G} \in \mathcal{G}$. For $x \in G$ we let $\hat{x}$ be an element in $\hat{G}$ with $\pi(\hat{x})=x$. We call $x$ doubling if $\hat{x}$ and $\hat{x} z$ are not conjugate in $\hat{G}$. Whether $x \in G$ is doubling or not is decided in $C_{G}(x)$. Indeed, let $\hat{C}_{G}(x):=\pi\left(C_{\hat{G}}(\hat{x})\right)$. Then if $C_{G}(x)=\hat{C}_{G}(x)$, then $x$ is doubling. Otherwise $x$ is not doubling and $\left|C_{G}(x): \hat{C}_{G}(x)\right|=2$. The doubling elements of $G$ form a union of conjugacy classes. The conjugacy classes of doubling elements are called doubling classes in $G$. A conjugacy class in $\hat{G}$ is called doubling, if its image under $\pi$ is doubling in $G$.

A spin character of $\hat{G}$ is an irreducible character which does not have $z$ in its kernel. The remaining irreducible characters of $\hat{G}$ are called linear.

Throughout this paper we assume that $p$ is an odd prime integer. Then each $p$-block of $\hat{G}$ contains only spin characters (a spin block) or only linear characters (a linear block).

For a subgroup $X$ of $G \in \mathcal{G}$ we let $\hat{X}:=\pi^{-1}(X)$.

When $\hat{G}_{1}, \hat{G}_{2} \in \mathcal{G}$, their twisted (central) product $\hat{G}_{1} \hat{\times} \hat{G}_{2}$ is defined as in 8 , p. 450]. Our main example of this occurs in the case where $G_{1}, G_{2}$ are subgroups of $S_{n}$ operating on disjoint sets, i.e., $G_{1}$ fixes the points moved by elements of $G_{2}$ and vice versa. Then $G_{1}$ and $G_{2}$ form a direct product as subgroups of $S_{n}$, and for the inverse images under $\pi$ we have $\widehat{G_{1} \times G_{2}}=\hat{G}_{1} \hat{\times} \hat{G}_{2}$.

We need that there is a surjective map $\hat{\otimes}$ associating to a pair of irreducible spin characters $\chi_{1}, \chi_{2}$ of $\hat{G}_{1}$ and $\hat{G}_{2}$ an irreducible spin character $\chi_{1} \hat{\otimes} \chi_{2}$ of $\hat{G_{1}} \hat{\times} \hat{G}_{2}$; see [11. Proposition 1.2] for the properties of this map. If $B_{1}$ and $B_{2}$ are spin blocks of $\hat{G}_{1}$ and $\hat{G}_{2}$, then

$$
B_{1} \hat{\otimes} B_{2}:=\left\{\chi_{1} \hat{\otimes} \chi_{2} \mid \chi_{1} \in B_{1}, \chi_{2} \in B_{2}\right\} .
$$

\section{BRAUER'S BLOCK SPLITTINGS}

There are different approaches to the theory of block splittings and lower defect group multiplicities $m_{B}(Q)$ or more generally the sectional lower defect group multiplicities $m_{B}^{(\sigma)}(Q)$, where $\sigma$ is a $p$-element, $Q$ a $p$-subgroup and $B$ a $p$-block of $G$. However, in this paper we consider only block splittings of the $p$-regular conjugacy classes, so $\sigma=1$. The basic idea is to split the conjugacy classes (or the conjugacy classes in a $p$-section determined by a $p$-element $\sigma$ ) into disjoint sets, one set for each block, such that certain conditions are fulfilled. (We call this a block splitting of the conjugacy classes.) When this is done, then the defect groups $Q$ of the 
conjugacy classes associated to a block $B$ are exactly the lower defect groups for $B$ and the number of occurrences of $Q$ is the lower defect group multiplicity $m_{B}(Q)$ or $m_{B}^{(\sigma)}(Q)$. For details we refer to 4 and $[12$. (See also [5.)

To begin with we need some results from [3, Section 5] and [4. Let $G$ be a finite group and $p$ a prime. Then $k(G)$ is the number of conjugacy classes (or the number of ordinary irreducible characters) of $G$. We denote by $C l_{p}(G)$ the set of $p$-regular conjugacy classes of $G$, and set $\ell(G)=\left|C l_{p}(G)\right|$. For a conjugacy class $C$ we take a representative $x_{C} \in C$. Furthermore, we denote by $\operatorname{Bl}(G)$ the set of $p$-blocks of $G$. If $B \in B l(G)$, then $\ell(B)$ denotes the number of modular irreducible characters in $B, \operatorname{Irr}(B)$ is the set of ordinary irreducible characters in $B$ and $k(B)$ its cardinality.

Brauer's results may (in part) be formulated as follows:

Theorem 2.1. There exists a set $L(G)$ of $\ell(G)$ irreducible characters of $G$ such that $\operatorname{det}\left(\chi\left(x_{C}\right)\right)_{\chi \in L(G), C \in C l_{p}(G)} \not \equiv 0 \bmod \wp$.

Furthermore, there exists a disjoint decomposition

$$
C l_{p}(G)=\bigcup_{B \in B l(G)} C l(B)
$$

and for each $B \in B l(G)$ a subset $L(B)$ of $L(G) \cap \operatorname{Irr}(B)$, such that for all $B \in B l(G)$ :

(1) $|L(B)|=|C l(B)|=\ell(B)$.

(2) $\operatorname{det}\left(\chi\left(x_{C}\right)\right)_{\chi \in L(B), C \in C l(B)} \not \equiv 0 \bmod \wp$.

Moreover, for each decomposition (*) satisfying (1) and (2) we have:

(3) For all $B \in B l(G)$ the elementary divisors of the Cartan matrix $C_{B}$ are exactly the orders of the p-defect groups of the conjugacy classes in $C l(B)$. More specifically, if $Q$ is a p-subgroup of $G$, then the number of conjugacy classes in $C l(B)$ with $Q$ as a defect group is exactly the lower defect group multiplicity $m_{B}^{(1)}(Q)$.

Given $L(G)$ as in the theorem, we define a block splitting of $C l_{p}(G)$ as a disjoint decomposition $(*)$ as above, such that for each $B \in B l(G)$ there exists a subset $L(B)$ of $L(G) \cap \operatorname{Irr}(B)$ satisfying (1) and (2) of the theorem.

Let $Q$ be a $p$-subgroup of $G$. For $N=N_{G}(Q), B l(N, B)$ is the set of $p$-blocks $b$ of $N$ with $b^{G}=B$. We have $m_{B}(1)=m_{B}^{(1)}(1)$, since the trivial group 1 can only be a defect group for $p$-regular conjugacy classes. In the following, some general formulas are needed. (See [4. (7E)] or [12, (6.3)] and [13, Lemma (3.8)].)

Lemma 2.2. Let $B$ be a p-block of a finite group $G$. Then:

(1) $\ell(B)-m_{B}(1)=\sum_{Q} m_{B}^{(1)}(Q)$, where $Q$ runs through a set of representatives for the conjugacy classes of non-trivial p-subgroups of $G$.

(2) $m_{B}^{(1)}(Q)=\sum_{b} m_{b}^{(1)}(Q)$, where $b$ runs through $\mathrm{Bl}\left(N_{G}(Q), B\right)$.

(3) The multiplicity of $p^{d}$ as an elementary divisor of the Cartan matrix of $B$ is $\sum_{Q} m_{B}^{(1)}(Q)$, where $Q$ runs through a set of representatives for the conjugacy classes of subgroups of order $p^{d}$ in $G$. 
We need also the following results for a group $\hat{G} \in \mathcal{G}$.

Proposition 2.3. Let $p$ be odd and take a set $L(\hat{G})$ of irreducible characters of $\hat{G} \in \mathcal{G}$ as in Theorem 2.1. Let $L_{l}(\hat{G})$ and $L_{s}(\hat{G})$ denote the subsets of linear characters and spin characters in $L(\hat{G})$, respectively. Then $C l_{p}(\hat{G})$ has a splitting $C l_{p}(\hat{G})=C l_{l} \cup C l_{s}$ such that $\operatorname{det}\left(\chi\left(x_{C}\right)\right)_{\chi \in L_{l}(\hat{G}), C \in C l_{l}} \not \equiv 0 \bmod \wp$ and $\operatorname{det}\left(\chi\left(x_{C}\right)\right)_{\chi \in L_{s}(\hat{G}), C \in C l_{s}} \not \equiv 0 \bmod \wp$.

In any such splitting, $\mathrm{Cl}_{l}$ contains all non-doubling p-regular classes of $\hat{G}$ and one of each pair of associated doubling p-regular classes in $\hat{G}$, while $C l_{s}$ contains the other of each pair of associated doubling p-regular classes.

This splitting can then be refined to a block splitting of the p-regular conjugacy classes of $\hat{G} \in \mathcal{G}$. Thus, in such a block splitting, each non-doubling p-regular class is associated to a linear block of $\hat{G}$, and for each doubling p-regular class in $G$ one of the classes in $\hat{G}$ is associated to a linear block and the other to a spin block of $\hat{G}$. In particular, all classes associated to spin blocks are doubling.

Proof. As in Brauer's proof of (5A) in [3] we consider a Laplacian expansion of the determinant $\operatorname{det}\left(\chi\left(x_{C}\right)\right)_{\chi \in L(G), C \in C l_{p}(G)}$ where we gather together the terms corresponding to linear and spin characters, respectively; each summand is then a product of a subdeterminant corresponding to the linear characters and a subdeterminant corresponding to the spin characters in $L(\hat{G})$. Hence there must be a splitting of the $p$-regular conjugacy classes into two subsets such that the corresponding subdeterminants both do not vanish mod $\wp$. As all spin characters vanish on all non-doubling classes in $\hat{G}$, these classes must all be associated to $L_{l}(\hat{G})$.

Now let $C$ be a doubling $p$-regular class in $G$, and $C_{+}$and $C_{-}$the corresponding classes in $\hat{G}$. By definition, any linear character of $\hat{G}$ has the same value on both classes $C_{+}$and $C_{-}$. Thus, these classes cannot both be associated to the linear characters, and at least one of the two classes has to be associated to $L_{s}(\hat{G})$. Now the number $\ell_{d}(G)$ of doubling $p$-regular conjugacy classes in $G$ is the number of $p$-modular irreducible spin representations of $\hat{G}$, and this is thus the maximum number of spin characters which are linearly independent $(\bmod \wp)$ on the $p$-regular classes. Thus the set $L_{s}(\hat{G})$ must contain exactly $\ell_{d}(G)$ spin characters, and for each pair of doubling $p$-regular conjugacy classes in $\hat{G}$ exactly one is associated to the spin characters.

By Brauer's argument (again expanding the determinant) this splitting may then be refined to a block splitting.

\section{SPIN BLOCKS AND DOUBLING CLASSES IN $\hat{S_{n}}$}

We need some notation for partitions. If $\lambda=\left(t_{1}, t_{2}, \ldots, t_{l}\right)$ is a partition of $n$, we write $\lambda \vdash n$ (or $|\lambda|=n$ ), the $t_{i}$ 's are the (non-zero) parts of $\lambda$ and the number $l$ of parts in $\lambda$ is called the length $l(\lambda)$ of $\lambda$. The $\operatorname{sign} \delta(\lambda)$ is defined as $(-1)^{n-l(\lambda)}$. Partitions with positive (negative) sign are called positive (negative). We primarily use the "exponential" notation for partitions $\lambda=\left(i^{a_{i}(\lambda)}\right)$ signifying that $\lambda$ has $a_{i}(\lambda)$ parts equal to $i$.

The labels of the doubling conjugacy classes of $S_{n}$ (the doubling partitions) were described in [16]. They are the partitions in $\mathcal{T}(n)=\mathcal{O}(n) \cup \mathcal{D}^{-}(n)$, i.e., an arbitrary partition with all parts odd or an odd partition with distinct parts. 
The structure of centralizers of elements in $S_{n}$ is well known. Let the element $x$ be contained in a conjugacy class labelled by the partition $\lambda \vdash n$. Thus $x$ contains $a_{i}(\lambda)$ disjoint cycles of length $i$ for each $i$ and the centralizer $C_{S_{n}}(x)$ is factored as a direct product of wreath products $\mathbb{Z}_{i}\left\langle S_{a_{i}(\lambda)}\right.$.

We write $\lambda \vdash_{p} n$ and call the partition $p$-class regular, if $a_{i}(\lambda)=0$ whenever $p \mid i$. Then the $p$-defect group of the $p$-regular conjugacy class labelled by a $p$-class regular partition $\lambda$ is isomorphic to the $p$-Sylow subgroup of the direct product $\prod S_{a_{i}(\lambda)}$. The $p$-Sylow subgroup of the symmetric group $S_{a}$ has order $p^{d_{p}(a)}$, where

$$
d_{p}(a)=\sum_{j \geq 1}\left[\frac{a}{p^{j}}\right] .
$$

Here [...] signifies "integral part of". Thus the $p$-defect of the class of $\lambda$ is given by

$$
d_{p}(\lambda)=\sum_{i, j \geq 1}\left[\frac{a_{i}(\lambda)}{p^{j}}\right] .
$$

If all parts of $\lambda$ are distinct, then $d_{p}(\lambda)=0$.

We have that $k\left(\hat{S}_{n}\right)-k\left(S_{n}\right)$ equals the number of doubling classes of $S_{n}$ and that $l\left(\hat{S}_{n}\right)-l\left(S_{n}\right)$ equals the number of $p$-regular doubling classes of $S_{n}$.

The spin characters of $\hat{S}_{n}$ are labelled by partitions in $\mathcal{D}(n)$ (and a sign). They are

$$
\left\{\langle\lambda\rangle \mid \lambda \in \mathcal{D}^{+}(n)\right\} \cup\left\{\langle\lambda\rangle_{ \pm} \mid \lambda \in \mathcal{D}^{-}(n)\right\}
$$

Here the characters labelled by partitions in $\mathcal{D}^{+}$are selfassociate, i.e., $\langle\lambda\rangle=\operatorname{sgn} \cdot\langle\lambda\rangle$, and the other characters are non-selfassociate; they are related via $\langle\lambda\rangle_{+}=\operatorname{sgn} \cdot\langle\lambda\rangle_{-}$. The following (often referred to as Morris' conjecture) was proved in [9], [7]:

Proposition 3.1. Let $\lambda, \mu \in \mathcal{D}(n)$.

(1) A spin character labelled by $\lambda$ is in a p-block of defect 0 if and only if $\lambda$ is a $\bar{p}$-core.

(2) If $\lambda$ is not a $\bar{p}$-core, then the characters labelled by $\lambda$ and $\mu$ are in the same spin block if and only if $\lambda$ and $\mu$ have the same $\bar{p}$-core.

This shows that a spin block $B$ has a well-defined core $\gamma(B)$, the $\bar{p}$-core of the partitions labelling the characters in $B$, and also a well-defined weight $w(B)=$ $(n-|\gamma(B)|) / p$. Based on this we define the sign $\delta(B)$ of $B$ by

$$
\delta(B):=(-1)^{w(B)} \delta(\gamma(B)) .
$$

It follows by an easy calculation that we also have $\delta(B)=(-1)^{n-l(\gamma(B))}$. Corresponding to its sign, we call the block a positive or negative spin block. By 15, this corresponds to all modular irreducible representations in $B$ being selfassociate or non-selfassociate, respectively. It should be remarked that this definition of the sign $\delta(B)$ of a spin block is different from the sign $\sigma(B)$ of a spin block defined in [13, section 3] and also used in [15] which was simply $\delta(\gamma(B))$. This change makes our formulas (generating functions) somewhat simpler. For details we refer to Section 5 .

In the following, the integer $t$ is defined by $t=(p-1) / 2$. The partitions $\lambda \in \mathcal{D}(n)$ having a given $\bar{p}$-core $\gamma \vdash n-w p$ are distinguished by their $\bar{p}$-quotients. They are $(t+1)$-tuples of partitions $\left(\lambda_{0}, \lambda_{1}, \ldots, \lambda_{t}\right)$ satisfying that $\lambda_{0} \in \mathcal{D}$ and $\left|\lambda_{0}\right|+\left|\lambda_{1}\right|+\cdots+$ $\left|\lambda_{t}\right|=w$. The corresponding generating function is $D(q) P(q)^{t}=P(q)^{t+1} / P\left(q^{2}\right)$, 
where $P(q)$ is the generating function for the number $p(n)$ of partitions of $n$ and $D(q)$ is the generating function for the number of partitions of $n$ into distinct parts.

We denote by $\mathcal{D}_{p}$ the set of $p$-class regular partitions in $\mathcal{D}$. It should be remarked that $\lambda \in \mathcal{D}_{p}$ exactly when the first partition $\lambda_{0}$ in the $\bar{p}$-quotient of $\lambda$ is empty. The corresponding generating function for the number of partitions $\lambda \in \mathcal{D}_{p}(n)$ having a given $\bar{p}$-core $\gamma \vdash n-w p$ is then $P(q)^{t}=\sum_{w>0} k(t, w) q^{w}$.

If $\langle\lambda\rangle \in B$ or $\langle\lambda\rangle_{ \pm} \in B$, then the signs of $\bar{\lambda}$ and $B$ are related by

$$
\delta(\lambda)=(-1)^{l\left(\lambda_{0}\right)} \delta(B)
$$

as is easily seen.

Lemma 3.2. Let $\lambda \in \mathcal{D}_{p}$.

(1) If $\mu$ is obtained from $\lambda$ by removing a p-bar, then $\mu \in \mathcal{D}_{p}$ and $\delta(\lambda) \neq \delta(\mu)$.

(2) Let $w=w(\lambda)$. Then $\delta(\lambda)=(-1)^{w} \delta\left(\lambda_{(\bar{p})}\right)$.

Proof. (1) As $\lambda \in \mathcal{D}_{p}$, we may remove a $p$-bar only in two ways. We either subtract $p$ from one of the parts, say $\lambda_{j}$, or we remove two parts $j$ and $p-j$, where $j \in$ $\{1, \ldots, t\}$. In the first case, the parity of $\lambda_{j}$ is changed, in the second case an even part is removed. Thus in both cases the parity of $\lambda$ is changed. The assertion in (2) now follows immediately.

From the results above we immediately obtain

Corollary 3.3. The spin characters $\langle\lambda\rangle, \lambda \in \mathcal{D}_{p}^{+}$, are in positive spin blocks, and the spin characters $\langle\lambda\rangle_{ \pm}, \lambda \in \mathcal{D}_{p}^{-}$, are in negative spin blocks.

Proposition 3.4. (1) Assume we are given a block splitting for the p-regular conjugacy classes of $\hat{S}_{n}$ as in Proposition 2.3. Let $B$ be a spin block, and let $C_{\lambda}$ be a conjugacy class of type $\lambda \in \mathcal{D}_{p}^{-}$associated to $B$. Then $B$ is a negative spin block.

(2) The number of $\mathcal{D}_{p}^{-}$-classes associated to a negative spin block of weight $w>0$ is $k(t, w)$.

Proof. (1) There are only two spin characters which are non-zero on $C_{\lambda}$, namely $\langle\lambda\rangle_{ \pm}$, hence one of these must be in $B$. Hence $B$ is a negative spin block. (2) follows then from the remarks on $\bar{p}$-quotients above.

Remark 3.5. In the case of two associate negative spin blocks of weight $w=0$, the corresponding $\mathcal{D}_{p}^{-}$-class is associated to one of the blocks, while an $\mathcal{O}_{p}$-class is associated to the other, where $\mathcal{O}_{p}$ denotes the $p$-class regular partitions in $\mathcal{O}$.

\section{A Reduction theorem for the multiplicity of 1}

In this section we study the multiplicity of 1 (the trivial $p$-subgroup) as a lower defect group in a spin block of $\hat{S}_{n}$. As mentioned earlier, only $p$-regular conjugacy classes can have 1 as a defect group, so for any $p$-block $B$ we have $m_{B}(1)=m_{B}^{(1)}(1)$. In order to compute $m_{B}(1)$ for a spin block we need to use Lemma 2.2, Our goal is to show that for a spin block $B, \ell(B)-m_{B}(1)$ depends only on the weight of $B$. By Lemma 2.2 (1) it suffices to show that for any $p$-subgroup $Q \neq 1$ the multiplicity $m_{B}^{(1)}(Q)$ depends only on the weight of $B$. We are thus led to study $\hat{N}$, where $N=N_{S_{n}}(Q)$. 
Whenever $Q$ is a $p$-subgroup of $S_{n}$, then the weight $w(Q)$ of $Q$ is defined as the integer $v$ such that $Q$ fixes exactly $n-v p$ points in $\{1,2, \ldots, n\}$. Thus the elements of $Q$ move a total of $v p$ points. It is not difficult to see the following lemma.

Lemma 4.1. Let $\lambda \in \mathcal{O}_{p}$. The weight of the defect group of the conjugacy class labelled by $\lambda$ is

$$
\sum_{i \geq 1} i\left[\frac{a_{i}(\lambda)}{p}\right]
$$

Lemma 4.2. Let $Q$ be a non-trivial p-subgroup of $S_{n}$ of weight $v$. We may also consider $Q$ as a p-subgroup of $\hat{S}_{n}$ (via the map $\pi$ ). Let $N=N_{S_{n}}(Q)$. We consider $Q$ as a subgroup of $S_{v p}$ and then $N=N_{0} \times N_{1}$, where $N_{0}=N_{S_{v p}}(Q)$ and $N_{1}=S_{n-v p}$. We have that $\hat{N}=\hat{N}_{0} \hat{\times} \hat{N}_{1}$. The group $\hat{N}_{0}$ has only one spin block $\hat{b}_{0}$.

Proof. It is clear that $N$ has a direct factorization as stated. Since $N_{0}$ and $N_{1}$ operate on disjoint sets, we get that $\hat{N}$ is as stated. Analogous to [13, Proposition (1.2)], we get that $\hat{N}_{0}$ has a unique spin block $\hat{b}_{0}$.

Using Cabanes' description of the subpairs for $\hat{S}_{n}[7$, Theorem A], we have

Lemma 4.3. Let $B$ be a spin block of weight $w$ and let $Q$ and $\hat{N}$ be as in the previous lemma. Let $\hat{b}_{1}$ be a spin block of $\hat{S}_{n-v p}$ with the same core as B. (This spin block is unique unless $v=w$, where $\hat{b}_{1}$ has defect 0 .) Then $B l(\hat{N}, B)$ contains the unique block $\hat{b}=\hat{b}_{0} \hat{\otimes} \hat{b}_{1}$.

We need to define a variation of the multiplicity numbers. Let $B$ be a spin block of weight $w$. For $w>0$ we then define $m_{B}^{+}(1)$ as $m_{B}(1)$, if $B$ is positive and as $m_{B}(1)-k(t, w(B))$, if $B$ is negative. For $w=0$ we define $m_{B}^{+}(1)=1$.

Lemma 4.4. Let the notation be as in Lemma 4.3. We have

$$
m_{B}^{(1)}(Q)=m_{\hat{b}}^{(1)}(Q)=m_{\hat{b}_{0}}^{(1)}(Q) m_{\hat{b}_{1}}^{+}(1) .
$$

Proof. The first equality follows from Lemma 2.2(2) and Lemma 4.3. We consider only $p$-regular classes throughout. We consider block splittings for $\hat{N}_{0}$ and $\hat{N}_{1}$ as in Section 2. The ( $p$-regular) conjugacy classes associated to $\hat{b}_{0}$ and $\hat{b}_{1}$ contain only doubling classes. By [12, Corollary (3.10)], we may restrict ourselves to conjugacy classes of $\hat{N}$ with $Q$ as a defect group. They are contained in the inverse images under $\pi$ of the conjugacy classes of $N$ with $Q$ as a defect group. These are products of conjugacy classes of $N_{0}$ with $Q$ as a defect group and conjugacy classes of $N_{1}$ of defect 0 . Conjugacy classes of $\hat{N}_{0}$ with $Q$ as a defect group are in bijection with the conjugacy classes of $\hat{S}_{p v}$ with $Q$ as a defect group. As $Q$ is non-trival, classes in $\mathcal{D}$ cannot occur. Thus the conjugacy classes of $\hat{N}_{0}$ in question are doubling, contain even permutations and they do not split in the relevant alternating group. To get doubling classes for $\hat{N}$ the conjugacy classes of $\hat{N}_{1}$ need to have the same property (see the proof of [8, Theorem 4.4]). Thus the $\mathcal{D}_{p}^{-}$-classes of $\hat{N}_{1}$ do not give a contribution. Note that these are only associated to the negative blocks (Proposition 3.4) and that in the exceptional case of two conjugate negative blocks of defect 0 , one of the two is associated to a $\mathcal{D}_{p}^{-}$-class. Hence by definition of $m_{\hat{b}_{1}}^{+}(1)$ we get in any case exactly $m_{\hat{b}_{1}}^{+}(1)$ classes of $\hat{N}_{1}$ associated to $\hat{b}_{1}$ giving a contribution. 
Theorem 4.5. Let $B$ be a spin block. Then $\ell(B)-m_{B}(1)$ and $m_{B}^{+}(1)$ depend only on the weight $w(B)$ of $B$.

Proof. The proof is by induction on the weight $w=w(B)$ of $B$, the result being trivially true for $w=0$ and also true for $w=1$ by Theorem (9.1) of [12. We use Lemma 2.2, and thus need to consider $m_{B}^{(1)}(Q)$, where $Q$ is a non-trivial $p$ subgroup of $\hat{S}_{n}$. Assume that $w(Q)=v>0$. Let $\hat{N}=N_{\hat{S}_{n}}(Q)$. By the previous three lemmas $\hat{N}$ is a twisted central product $\hat{N}=\hat{N}_{0} \hat{\times} \hat{N}_{1}$, where $N_{0}=N_{S_{v p}}(Q)$ and $N_{1}=S(n-v p)$. Moreover, $\hat{N}_{0}$ has only one spin block $\hat{b}_{0}$. If $\hat{b}_{1}$ is the spin block of $\hat{S}_{n-p v}$ with the same core as $B$, then $m_{\hat{B}}^{(1)}(Q)=m_{\hat{b}_{0}}^{(1)}(Q) m_{\hat{b}_{1}}^{+}(1)$. Now $m_{\hat{b}_{0}}^{(1)}(Q)$ equals the number $\alpha_{Q}(v)$ of doubling $p$-regular conjugacy classes of $\hat{S}_{v p}$ with $Q$ as a defect group. By Lemma 2.2 (2) we now get

$$
\ell(B)-m_{B}(1)=\sum_{v>0} \alpha(v) m_{\hat{b}_{1}}^{+}(1),
$$

where $\alpha(v)$ is the number of doubling $p$-regular conjugacy classes of $S_{v p}$ with defect groups of weight $v$. Since $v>0$ and $\hat{b}_{1}$ has weight $w-v$, we get that $m_{\hat{b}_{1}}^{+}(1)$ is independent of the sign of $\hat{b}_{1}$ and thus $x(B):=\ell(B)-m_{B}(1)$ depends only on $w$. In both cases ( $B$ positive or negative) $m_{B}^{+}(1)=k(t, w)-x(B)$. Thus also $m_{B}^{+}(1)$ depends only on the weight.

We have now seen that the multiplicity $m_{B}^{+}(1)$ for a spin block $B$ depends only on $w=w(B)$. We denote this multiplicity simply by $m^{+}(w)$. Thus the formula of Lemma 4.4 now reads

$$
m_{B}^{(1)}(Q)=\alpha_{Q}(w(Q)) m^{+}(w(B)-w(Q)) .
$$

Remark. Formula (11) may be generalized to arbitrary sectional multiplicities $m_{B}^{(\sigma)}(Q), \sigma$ a $p$-element not necessarily 1 , as follows:

$$
m_{B}^{(\sigma)}(Q)=\alpha_{Q}^{(\sigma)}(w(Q)) m^{+}(w(B)-w(Q)),
$$

where $\alpha_{Q}^{(\sigma)}(w(Q))$ is the number of doubling conjugacy classes in the $p$-section determined by $\sigma$ (inside $\hat{S}_{p w(Q)}$ ) with $Q$ as a defect group.

As a consequence of formula (1) we get one of our main results, confirming, in particular, a conjecture made by Brundan and Kleshchev in [6] that the determinants of the spin Cartan matrices only depend on the weight.

Theorem 4.6. The Cartan matrices for spin blocks of the same weight have the same elementary divisors $\neq 1$. In particular, the determinant of the Cartan matrix $C_{B}$ of a spin block $B$ depends only on the weight $w=w(B)$.

Proof. In case $w=0$ we have blocks of defect 0 and there is nothing to prove. Let $B$ be a spin block of weight $w>0$ of $\hat{S}_{n}$. By Lemma 2.2 (3) the multiplicity of $p^{d}$ as an elementary divisor in the Cartan matrix $C_{B}$ of $B$ equals $\sum_{\left\{Q|| Q \mid=p^{d}\right\}} m_{B}^{(1)}(Q)$. Moreover, if $Q$ is a $p$-subgroup of positive weight $v \leq w$, then by Theorem 4.5 $m_{B}^{(1)}(Q)=\hat{m}(Q) m^{+}(w-v)$, where $\hat{m}(Q)$ is the number of doubling $p$-regular conjugacy classes of $\hat{S}_{p v}$ with $Q$ as a defect group. If $v>w$, the multiplicity $m_{B}^{(1)}(Q)$ is 0 . Thus all multiplicities of elementary divisors $\neq 1$ in $B$ are independent of the core and sign of $B$, proving the theorem. 
It should be noted that an explicit formula for the determinant of $C_{B}$ may be obtained using Theorem 5.6 below; this provides an alternative proof for the formula given in [6].

\section{EXPLICIT FORMULAS FOR MULTIPLICITIES AND DETERMINANTS}

In this section we compute a number of generating functions along the lines of [13] and 2, from which the (regular) multiplicities of lower defect groups for spin blocks may be computed and also the elementary divisors and the determinant of their Cartan matrices. This is possible because, as we have seen, these numbers only depend on the weight and the sign of the spin block.

We start with the generating functions $K^{\delta}(q), L^{\delta}(q)$ and $M^{\delta}(q)$ for (respectively) the number of ordinary irreducible characters, the number of modular irreducible characters and the multiplicity of 1 as an elementary divisor of the Cartan matrix for a spin block of weight $w$ and sign $\delta$. The two former were computed in [14] and [15], but are included here for completeness and because the definition of the sign of a spin block is different here.

As before, $P(q)$ is the generating function for the number of partitions and $t=(p-1) / 2$.

Proposition 5.1. Let $B$ be a spin block of $\hat{S}_{n}$ of weight $w$ and sign $\delta$. Then the numbers $k(B)$ and $\ell(B)$ of ordinary and modular spin characters in $B$ depend only on $w$ and $\delta$. If we denote $k(B)=k^{\delta}(\bar{p}, w), \ell(B)=\ell^{\delta}(\bar{p}, w)$, then the corresponding generating functions are

$$
\begin{gathered}
K^{\delta}(q)=\frac{1}{2} P(q)^{t-1}\left(3 \frac{P(q)^{2}}{P\left(q^{2}\right)}-\delta\right), \\
L^{+}(q)=P(q)^{t}, L^{-}(q)=2 P(q)^{t} .
\end{gathered}
$$

In particular,

$$
K^{-}(q)-K^{+}(q)=P(q)^{t-1}
$$

and

$$
L^{-}(q)-L^{+}(q)=P(q)^{t}
$$

In view of the results of Section 4 we have also for the multiplicities of 1 as an elementary divisor that

$$
M^{-}(q)-M^{+}(q)=P(q)^{t}
$$

in analogy with the last part of the above proposition. Also, $M^{+}(q)=$ $\sum_{w \geq 0} m^{+}(w) q^{w}$, where $m^{+}(w)$ is as defined in Section 4. We proceed to compute a formula for $M^{+}(q)$. First we note a simple consequence of Lemma 4.1

Lemma 5.2. Let $v \geq 1$. The number $\alpha(v)$ of p-regular doubling classes of $\hat{S}_{p v}$ with a defect group of weight $v$ equals $\left|\mathcal{O}_{p}(v)\right|$.

The generating function for $\left|\mathcal{O}_{p}(w)\right|$ is easily seen to be

$$
O_{p}(q)=\frac{P(q) P\left(q^{2 p}\right)}{P\left(q^{2}\right) P\left(q^{p}\right)} .
$$


We can now show:

Theorem 5.3. The generating function $M^{+}(q)$ for the multiplicity of 1 as an elementary divisor in positive spin blocks is

$$
M^{+}(q)=\frac{P(q)^{t}}{O_{p}(q)}=\frac{P(q)^{t-1} P\left(q^{2}\right) P\left(q^{p}\right)}{P\left(q^{2 p}\right)} .
$$

The corresponding generating function $M^{-}(q)$ for negative spin blocks is given by

$$
M^{-}(q)=M^{+}(q)+P(q)^{t} .
$$

Proof. Set $\alpha(0)=1$. Proposition 5.1 and the proof of Theorem 4.5 show that if $B$ is a positive spin block of weight $w$, then

$$
\ell(B)=k(t, w)=\sum_{v \geq 0} \alpha(v) m^{+}(w-v) .
$$

For the generating functions this means that

$$
P(q)^{t}=O_{p}(q) M^{+}(q),
$$

proving the first statement. Then apply equation (2) to get the second statement.

Recall that for a $p$-class regular partition $\lambda$ its $p$-defect is given by

$$
d_{p}(\lambda)=\sum_{i, j \geq 1}\left[\frac{a_{i}(\lambda)}{p^{j}}\right] .
$$

We put $e_{p}(\lambda)=d_{p}(\lambda)+l(\lambda)$.

Let $\lambda \in \mathcal{O}_{p}(n)$. Write for each $i \geq 1 a_{i}(\lambda)=m_{i}(\lambda) p+r_{i}(\lambda)$, where $0 \leq$ $r_{i}(\lambda) \leq p-1$. Notice that $m_{i}(\lambda)=\left[\frac{a_{i}(\lambda)}{p}\right]$. Let $\mu=\left(1^{m_{1}(\lambda)} 3^{m_{3}(\lambda)} \ldots\right)$ and $\rho=\left(1^{r_{1}(\lambda)} 3^{r_{3}(\lambda)} \ldots\right)$. Clearly, $\mu, \rho \in \mathcal{O}_{p}$ and $\rho$ is of defect 0 . Moreover, $|\mu| p+|\rho|=n$ and $\lambda$ is uniquely determined by $\mu$ and $\rho$. Now since $a_{i}(\mu)=m_{i}(\lambda)$ for all $i$, we have

$$
\begin{aligned}
d_{p}(\lambda) & =\sum_{i, j \geq 1}\left[\frac{a_{i}(\lambda)}{p^{j}}\right]=\sum_{i, j \geq 1}\left[\frac{m_{i}(\lambda) p}{p^{j}}\right] \\
& =\sum_{i, j \geq 1}\left[\frac{a_{i}(\mu)}{p^{j}}\right]+\sum_{i \geq 1} a_{i}(\mu)=d_{p}(\mu)+l(\mu)=e_{p}(\mu),
\end{aligned}
$$

which is independent of $\rho$.

For the defect groups we have that if $\mu^{*}$ is defined by $a_{i}\left(\mu^{*}\right)=p a_{i}(\mu)=p\left[\frac{a_{i}(\lambda)}{p}\right]$, then the conjugacy classes labelled by $\lambda \in \mathcal{O}_{p}(n)$ and $\mu^{*} \in \mathcal{O}_{p}(p|\mu|)$ have isomorphic defect groups of weight $|\mu|$.

For $i \geq 1$ let

$$
f_{i}(v)=\left|\left\{\mu \in \mathcal{O}_{p}(v) \mid e_{p}(\mu)=i\right\}\right| .
$$

Then we can show

Theorem 5.4. Let $i \geq 1$. The multiplicity of $p^{i}$ as an elementary divisor of the Cartan matrix $C_{B}$ of a spin block $B$ of weight $w \geq 1$ is

$$
r_{i}(w)=\sum_{v \geq 1} f_{i}(v) m^{+}(w-v) .
$$


Proof. By Lemma 4.4 and Theorem 4.5 we have for a $p$-subgroup $Q$ of $S_{n}$ of weight $v$ that $m_{B}^{(1)}(Q)=f(Q) m^{+}(w-v)$, where $f(Q)$ is the number of doubling classes of $\hat{S}_{p v}$ with $Q$ as a defect group. Thus by Lemma 2.2 (3) we get that $r_{i}(w)=$ $\sum_{|Q|=p^{i}} f(Q) m^{+}(w-w(Q))$ (where the sum is over a set of representatives of the conjugacy classes of subgroups with the given property). Thus we need to show for $v \geq 1$ that

$$
\sum_{\left\{Q|w(Q)=v,| Q \mid=p^{i}\right\}} f(Q)=f_{i}(v) .
$$

But this is an immediate consequence of the discussion preceding this result.

Put $s_{p}(n)=\sum_{\lambda \in \mathcal{O}_{p}(n)} d_{p}(\lambda)$. Then by Theorem 2.1 the determinant of the spin Cartan matrix of $\hat{S}_{n}$ is $p^{s_{p}(n)}$. In addition, let $u_{p}(n)=\sum_{\lambda \in \mathcal{O}_{p}(n)} l(\lambda)$ and let $z_{p}(n)$ be the number of partitions in $\mathcal{O}_{p}(n)$ of defect 0 .

If we sort the partitions $\lambda \in \mathcal{O}_{p}(n)$ according to the size $v$ of $\mu$ (as above) we obtain

$$
\begin{aligned}
s_{p}(n) & =\sum_{\lambda \in \mathcal{O}_{p}(n)} d_{p}(\lambda)=\sum_{v \geq 1}\left(\sum_{\mu \in \mathcal{O}_{p}(v)} e_{p}(\mu)\right) z_{p}(n-p v) \\
& =\sum_{v \geq 1}\left(s_{p}(v)+u_{p}(v)\right) z_{p}(n-p v)
\end{aligned}
$$

For the corresponding generating functions $S_{p}(q), U_{p}(q), Z_{p}(q)$ this means

$$
S_{p}(q)=\left(S_{p}\left(q^{p}\right)+U_{p}\left(q^{p}\right)\right) Z_{p}(q) .
$$

It is fairly straightforward to find expressions for $Z_{p}(q)$ and $U_{p}(q)$, along the lines of [2]. Indeed,

$$
Z_{p}(q)=\frac{O_{p}(q)}{O_{p}\left(q^{p}\right)}=\frac{P(q) P\left(q^{2 p}\right)^{2} P\left(q^{p^{2}}\right)}{P\left(q^{p}\right)^{2} P\left(q^{2 p^{2}}\right) P\left(q^{2}\right)} .
$$

The generating function $T(q)$ for the number $t(n)$ of divisors of $n$ may be expressed as follows:

$$
T(q)=\sum_{i \geq 1}\left(q^{i}+q^{2 i}+\ldots\right)=\sum_{i \geq 1} \frac{q^{i}}{1-q^{i}} .
$$

Let $T_{2}(q)=T(q)-T\left(q^{2}\right)$, the generating function for the number of odd divisors of $n$, and set $T_{p}^{*}(q)=T_{2}(q)-T_{2}\left(q^{p}\right)$, the generating function for the number $t_{p}^{*}(n)$ of odd divisors of $n$ prime to $p$. Arguing as in [2, Proposition 2.2] we obtain

$$
U_{p}(q)=O_{p}(q) T_{p}^{*}(q) .
$$

We can now prove

Theorem 5.5. The generating function for the exponent $s_{p}(n)$ of the determinant of the spin Cartan matrix of $\hat{S}_{n}$ is

$$
S_{p}(q)=O_{p}(q) T_{2}\left(q^{p}\right) .
$$

Proof. Define $S_{p}^{o}(q)=S_{p}(q) / O_{p}(q)$. To prove the theorem we need to show that $S_{p}^{o}(q)=T_{2}\left(q^{p}\right)$.

We divide equation (3) by $O_{p}(q)$ and apply equations (4) and (5) to get

$$
S_{p}^{o}(q)=\frac{S_{p}\left(q^{p}\right)+U_{p}\left(q^{p}\right)}{O_{p}\left(q^{p}\right)}=S_{p}^{o}\left(q^{p}\right)+T_{p}^{*}\left(q^{p}\right) .
$$


Iterating this we obtain

$$
S_{p}^{o}(q)=\sum_{j \geq 1} T_{p}^{*}\left(q^{p^{j}}\right)=T_{2}\left(q^{p}\right)
$$

proving the proposition.

In view of Theorem 4.6 we may now easily prove:

Theorem 5.6. The generating function for the exponent $c_{p}(w)$ of the determinant of the Cartan matrix of a spin block of weight $w$ in $\hat{S}_{n}$ is

$$
C_{p}(q)=P(q)^{t} T_{2}(q) .
$$

Proof. If $r_{p}(n)$ is the number of $\bar{p}$-cores of $n$, then by Proposition 3.1 there are, for $w \geq 1$, exactly $r_{p}(n-p w)$ spin blocks of $\hat{S}_{n}$ of weight $w$. Note that spin blocks of weight $w=0$ also have defect 0 and thus have a Cartan matrix determinant $p^{0}=1$. Thus for the exponents of Cartan matrix determinants we obtain

$$
s_{p}(n)=\sum_{w \geq 1} c_{p}(w) r_{p}(n-p w)
$$

or for the corresponding generating functions

$$
S_{p}(q)=C_{p}\left(q^{p}\right) R_{p}(q) .
$$

As noticed before, the arithmetic of the $p$-bar abacus implies that the generating function $R_{p}(q)$ satisfies

$$
R_{p}(q) P\left(q^{p}\right)^{t}=D_{p}(q),
$$

where $D_{p}(q)$ is the generating function for the number of $p$-class regular partitions in $\mathcal{D}_{p}$. As $\left|\mathcal{D}_{p}(n)\right|=\left|\mathcal{O}_{p}(n)\right|$ for all $n$, we thus have

$$
R_{p}(q)=\frac{O_{p}(q)}{P\left(q^{p}\right)^{t}} .
$$

The assertion now follows easily from equation (6) and Theorem 5.5 .

Note that since $T_{2}(q)=T(q)-T\left(q^{2}\right)$, the formula above agrees with the generating function computed by Brundan and Kleshchev in [6] (in their article, this was only obtained for positive spin blocks).

\section{ACKNOWLEDGMENT}

The second author wishes to thank the Department of Mathematics of the University of Auckland and especially Professor Jianbei An for the hospitality during his stay there in 2003.

\section{REFERENCES}

[1] G.E. Andrews, The theory of partitions, Addison-Wesley, 1976 (Reprinted, Cambridge University Press, 1998). MR1634067 (99c:11126)

[2] C. Bessenrodt, J. B. Olsson, A note on Cartan matrices for symmetric groups, Arch. Math. 81 (2003), 497-504. MR.2029709 (2004h:20014)

[3] R. Brauer, Zur Darstellungstheorie der Gruppen endlicher Ordnung, Math. Z. 63 (1956), 406444. (Reprinted in Collected works, vol. 2, 22-60, MIT Press, 1980.) MR0075953 (17:824g)

[4] R. Brauer, Defect groups in the theory of representations of finite groups. Illinois J. Math. 13 (1969), 53-73. (Reprinted in Collected works, vol. 2, 566-586, MIT Press, 1980.) MR0246979 $(40: 248)$ 
[5] M. Broué, Brauer coefficients of $p$-subgroups associated with a $p$-block of a finite group, $J$. Algebra 56 (1979), 365-383. MR0528581 (80d:20013)

[6] J. Brundan, A. Kleshchev, Cartan determinants and Shapovalov forms, Math. Ann. 324 (2002), 431-449. MR1938453(2003j:17033)

[7] M. Cabanes, Local structure of the p-blocks of $\tilde{S}_{n}$, Math. Z. 198 (1988), 519-543. MR 0950581 (89g:20020)

[8] J. F. Humphreys, On certain projective modular representations of direct products. J. London Math. Soc. 32 (1985), 449-459. MR0825920 (87g:20013)

[9] J. F. Humphreys, Blocks of projective representations of the symmetric groups, J. London Math. Soc. (2) 33 (1986), 441-452. MR0850960 (87k:20027)

[10] G. James, A. Kerber, The representation theory of the symmetric group, Addison-Wesley, 1981. MR0644144 (83k:20003)

[11] G. Michler, J. B. Olsson, The Alperin-McKay conjecture holds in the covering groups of symmetric and alternating groups, $p \neq 2, J$. Reine Angew. Math. 405 (1990), 78-111. MR 1040996 (91b:20020)

[12] J. B. Olsson, Lower defect groups, Comm. Algebra 8 (1980), 261-288. MR0558114(81g:20024)

[13] J. B. Olsson, Lower defect groups in symmetric groups, J. Algebra 104 (1986), 37-56. MR $865885(87 \mathrm{~m}: 20039)$

[14] J. B. Olsson, On the $p$-blocks of symmetric and alternating groups and their covering groups. J. Algebra 128 (1990), 188-213. MR:1031917 (90k:20022)

[15] J. B. Olsson, The number of modular characters in certain p-blocks. Proc. London Math. Soc. (3) 65 (1992), 245-264. MR1168188 (93e:20019)

[16] I. Schur, Über die Darstellung der symmetrischen und der alternierenden Gruppe durch gebrochene lineare Substitutionen. J. Reine Angew. Math. 139 (1911), 155-250. (Reprinted in Gesammelte Abhandlungen, Band I, 346-441, Springer, Berlin, 1973.) Translation: I. Schur, On the representation of the symmetric and alternating groups by fractional linear substitutions. (Translated from the German by Marc-Felix Otto.) Internat. J. Theoret. Phys. 40 (2001), 413-458. MR.1820589 (2003a:20016)

Institut für Mathematik, Universität Hannover, D-30167 Hannover, Germany

E-mail address: bessen@math.uni-hannover.de

Matematisk Afdeling, University of Copenhagen, Copenhagen, Denmark

E-mail address: olsson@math.ku.dk 